\title{
A Compact Dual-Band UHF Microstrip Patch Antenna for CubeSat Applications
}

\author{
Wellington G. da Silva, Antonio L. P. S. Campos, and Juan R. F. Guerra
}

\begin{abstract}
This paper presents a slitted, dual-band, square, microstrip patch antenna for CubeSat application. Compared to recently reported works, the originality of this design is the capability to operate at dual-band UHF, using a single feeding point, and, in this way, reducing the risks inherent to wire antennas. The antenna resonates at $399.025 \mathrm{MHz}$ and 431.600 MHz frequencies and it is limited at $100 \mathrm{~mm} \times 100 \mathrm{~mm}$ area in a CubeSat face. Forty slits, on the perimeter of a square patch, ten slits on each edge, and a high relative permittivity substrate was used to decrease the operating frequencies. An asymmetry between the slits allows the operation in two different frequencies. This slitted square patch may be up to $48 \%$ smaller in area than a square patch without slits, both with the same operating frequency. The design, simulation, manufacturing, and measurement details are presented. Simulated and measured results reveal that the gain, the radiation patterns, the bandwidth, and other parameters are adequate for CubeSat applications.
\end{abstract}

Index Terms-Compact antenna, Cubesat, Microstrip Patch Antenna, Miniaturization

\section{INTRODUCTION}

The CubeSat standard was developed to facilitate access to space for undergraduate students. This standard specifies a modular structure based on CubeSat unit (1U), which is a cube with the side equal to $100 \mathrm{~mm}$, according to Fig. 1, [1]. CubeSat satellites require antennas to realize telemetry, tracking and command (TT\&C); downlink for payload data; GPS/GNSS signal reception; and inter-satellite cross links. The area available in the smallest CubeSat for the antenna allocation is $100 \mathrm{~mm} \times 100 \mathrm{~mm} \mathrm{[1],} \mathrm{[2].} \mathrm{Therefore,} \mathrm{these}$ antennas should be small size and light. The most common solution for VHF-UHF antennas in CubeSat applications is the linear wire deployable antennas, specially dipole antennas and their variations. However, mechanical deployment may raise the possibility of mission failure. Several missions employing small satellites have failed because of these mechanical devices [3]. Many CubeSat operates in VHF (144 - $146 \mathrm{MHz})$ and UHF (435 - $438 \mathrm{MHz}$ ) frequency bands.

The aim of this work is to design, simulate, build and measure a dual-band UHF microstrip patch antenna for application in the data-uplink (400 - $402 \mathrm{MHz}$ ) and in the command uplink (430 - $440 \mathrm{MHz}$ ). These frequencies are chosen according to CONASAT Brazilian Satellites project, under development by INPE (National Institute for Space Research) in partnership with the AEB (Brazilian Space Agency). In accordance with

W. G. da Silva (wellington.silva@ufrn.edu.br), A. L. P. S. Campos (alpscampos@gmail.com) and J. R. F. Guerra (juanfguerra@ufrn.edu.br) are with Federal University of Rio Grande do Norte, Natal - Rio Grande do Norte Brazil.

Digital Object Identifier: 10.14209/jcis.2021.18 the preliminary design document, CONASAT constellation satellites will operate at the frequencies presented in Table I [4].



Fig. 1. Standard $1 \mathrm{U}$ CubeSat.

TABLE I

FREQUENCIES PLANNED FOR CONASAT SATELLITES, ADAPTED FROM [4]

\begin{tabular}{lcc}
\hline Subsystem & Frequency (MHz) & Antenna Type \\
\hline Command uplink & $430-440$ & Wired dipole \\
Telemetry downlink & 145.865 & Wired dipole \\
Data uplink & 401.620 & Wired dipole \\
Data downlink & 2267.520 & Microstrip \\
\hline
\end{tabular}

Compared with recently reported works, the originality of this work is the capability to operate at dual band with a single feed point, and, in this way, to decrease the risks inherent to wire antennas besides others advantages [5]. In the UHF and VHF band, the wavelength is increased resulting in relatively large antennas. Therefore, it is necessary to employ antenna miniaturization techniques [6]. These techniques can be classified into six categories [7], [8]:

1) loading the antenna with high-contrast material, high permittivity, and/or high permeability.

2) modifying and optimizing the antenna shape.

3) using lumped components to compensate for the reactive impedance. 
4) using artificially engineered electromagnetic metamaterials.

5) using shorting post; and

6) using an optimization method (fuzzy logic, genetic algorithm...).

Antenna size reduction is restricted by fundamental physical limits, in terms of a trade-off between radiation performances and impedance bandwidth [9]. Therefore, ensuring antenna performance is the main challenge when designing small antennas [10]. Wheeler proposed the electrically small antenna, or ESAs, defined as an antenna whose dimensions are much less than the wavelength $\lambda$ and it is subject to limitations [11]. The small antenna to be considered is one whose maximum dimension is less than the radianlength defined by $\lambda / 2 \pi$.

The minimum circumscribing sphere surrounding the antenna of maximum dimension $2 a$ is known as Chu's sphere [8], [12]. The $k a$ product is generally used as a synonym for the electrical size for small antennas, where $k$ is the wavenumber $2 \pi / \lambda$ [8]. The small antenna performance is limited by their electrical size $(k a)$, fractional bandwidth (FBW), quality factor $(Q)$, and gain $(G)$ [11]. Therefore, understanding these parameters is important. Of particular interest is how the antenna size is related to the bandwidth (or $Q$ ) [8]. Wheeler noted that antenna size limits radiation resistance values, efficiency, and bandwidth. In other words, antenna design is a quality factor $(Q)$, among volume, bandwidth, gain, and efficiency [11]. The best trade-off is usually attained when most of the available volume is excited by radiation [8].

Size, efficiency (gain), and bandwidth are highly interrelated and cannot be simultaneously optimized. That is, antenna size is reduced at the expense of decreased bandwidth or decreased efficiency, or both [7]. Furthermore, a perfect impedance matching between an antenna and a transmission line is not a trivial task [13]. In addition to limited bandwidth, small antennas also suffer from low radiation efficiency. This is because the radiation resistance decays rapidly in relation to the loss resistance [7]. Harrington extended the Wheeler and Chu works, on small antennas, including the loss effect. Harrington showed that the antenna maximum gain could be estimated using 1 [14].

$$
G_{\max }=(k a)^{2}+2 k a
$$

Radiation efficiency can be improved by reducing losses on the antenna by, for example, using planar strips instead of thin wires. Radiation resistance can be increased by filling more of the volume (inside of the Chu sphere) with antenna structure [7]. Some authors have presented papers addressing UHF antennas for small satellites applications, as presented in the following section.

\section{Antennas for CubeS ats: Literature Review}

Klofas did one of the early reviews on CubeSat antennas, he included the CubeSats launched from 2003 to 2008 [15]. Later Klofas published complementary research involving launches from 2009 to 2012 [16]. More recently, Kakoyiannis [17], Lokman [10], and Samii [18] have published reviews on the subject. This literature review showed wire antennas elements like linear monopoles or dipoles antennas are the most common solution for nanosatellite missions at VHF-UHF bands, despite increasing the risk of failure. However, to this date, in the literature, there are only a few studies on the use of the microstrip patch antennas operating in VHF-UHF for CubeSat. Between these works, we have highlighted some proposals.

Huang (2001) developed a compact UHF microstrip patch antenna for a possible Mars mission [19]. The proposal is a linearly polarized dual-band circular patch antenna with two feed point using coaxial probes and four slits with different length. The lowest frequency at $401.5 \mathrm{MHz}$ is obtained by the interaction of one feed point with the longest slits, while the other feed point combined with the shortest slits produces the highest frequencies at $437.1 \mathrm{MHz}$. The author designed a $120 \mathrm{~mm}$ diameter circular microstrip patch, using a $7.6 \mathrm{~mm}$ thickness substrate. A 9.8 relative permittivity Rogers TMM was used, the total antenna weight was 250 grams. The author claims that the antenna presented a gain of $1.0 \mathrm{dBi}$, directivity of $1.8 \mathrm{dBi}$ and efficiency of $83 \%$. These parameters are very good compared with a compact antenna. The prototype presented a bandwidth of $1.2 \mathrm{MHz}$ and $1.4 \mathrm{MHz}$ at 401.5 $\mathrm{MHz}$ and $437.1 \mathrm{MHz}$, respectively. Therefore, it is close to $0.3 \%$ of relative bandwidth. Although not being an antenna designed for a CubeSat application, this work is interesting for our research because it presents an antenna with dimensions and operating frequencies compatible with the demands of the CONASAT Project [4].

Kakoyiannis (2011) proposed a microstrip patch antenna by etching ten slits on each of the four sides of the square patch Kakoyiannis [17]. Theoretically, this tapering would force the current to go through the center of the patch, and thus produce an effective physical length. The antenna resonated near 436.5 $\mathrm{MHz}$ showing a bandwidth of $2 \mathrm{MHz}$, a gain of $-1.2 \mathrm{~dB}$, and a radiation efficiency of $52 \%$. A quarter-wave impedance transformer was used to match the peripheral impedance of the square microstrip patch with that of the $50 \Omega$ microstrip line feed. However, this proposal has the disadvantage of occupying an area of $120 \mathrm{~mm} \times 170 \mathrm{~mm}$, being incompatible with the restriction of $100 \mathrm{~mm} \times 100 \mathrm{~mm}$.

In the most recent work, Alam (2019) proposed an EMNZ (Epsilon-and-mu-near-zero) metamaterial inspired antenna and has attained measured bandwidth $\left(\left|S_{11}\right|<10 \mathrm{~dB}\right)$ of 14.92 $\mathrm{MHz}(391.00 \mathrm{MHz}-405.92 \mathrm{MHz})$. The perceptible originality of this proposal is the employment of metamaterial that significantly enhances the UHF antenna operating frequency stability, furthermore, this approach increases the efficiency for small satellites. The occupied area was printed patch 80 $\mathrm{mm} \times 45 \mathrm{~mm} \times 1.575 \mathrm{~mm}$. The obtained gain was $-1.15 \mathrm{~dB}$ with an omnidirectional radiation pattern [3].

\section{Methodology}

Starting with the analysis of previous works, a slitted microstrip patch antenna was designed to obtain a dual-band antenna operating at $401-402 \mathrm{MHz}$ and $430-440 \mathrm{MHz}$ occupying an area as small as $100 \mathrm{~mm}$ x $100 \mathrm{~mm}$. Simulated results presented within this work were determined using Ansys HFSS (High-Frequency Structural Simulator) software. 
We used a vacuum box with radiation boundaries to enclose the antenna. For better accuracy, the distance between the radiation boundary in each direction and the radiator must be not less than 1/4 wavelength. Therefore, the dimensions of the vacuum box were set to $550 \mathrm{~mm} \times 550 \mathrm{~mm}$ x $400 \mathrm{~mm}$ (X, Y, Z dimensions). A circular sheet was defined as a wave excitation port placed between the inner conductor (pin) and the outer conductor (shield) of the coaxial cable.

After obtaining satisfactory simulated results, a prototype antenna was built and measured. Two miniaturization techniques are combined to obtain a high degree of miniaturization of a microstrip patch antenna. The first technique is the use of a high permittivity substrate, and the second one is to load the patch with a slitted geometry.

The choice of the substrate must consider a suitable material of appropriate thickness, dielectric constant, and loss tangent. A thicker substrate, besides being mechanically strong, will increase the radiated power and improve impedance bandwidth. The fringing effect governs the antenna's radiation behavior. In this aspect, increasing the thickness of the dielectric increases the fringing effect and thus the radiated power [20]. However, it will also increase the weight, dielectric loss, surface waves loss, and extraneous radiations from the probe feed [20], [21]. The substrate permittivity $\epsilon$ plays a role similar to that of substrate thickness. A low value of $\epsilon$ for the substrate will increase the fringing field at patch periphery, and thus the radiated power. Therefore, substrates with $\epsilon$ less than or equal to 2.5 are preferred unless a smaller patch size is desired.

An increase in the substrate thickness has a similar effect on antenna characteristics as a decrease in the value of $\epsilon$ [20]. A high loss tangent increases dielectric loss and therefore reduces antenna efficiency [20]. The Fig. 2 shows the behavior of impedance and efficiency as a function of the thickness $d$ of the substrate as computed using a cavity model. Although a thick substrate improves the impedance bandwidth, decreases the efficiency. However, this approach only includes power lost due to surface waves and neglects dielectric and conductor losses [21].



Fig. 2. Impedance bandwidth $(S W R \leq 2)$ and efficiency for a square microstrip antenna element versus substrate thickness [21].
For an operating frequency of $430 \mathrm{MHz}$ and a substrate thickness (d) of $6.35 \mathrm{~mm}$, the $\mathrm{d} / \lambda$ ratio is approximately 0.01 . Therefore, considering (2), for a substrate with a relative permittivity of 10 , efficiency of the order of $85 \%$, and a bandwidth close to $1 \%$ are expected.

In addition to the radiation parameters, it is necessary to consider the spatial environment when choosing the substrate material. Extreme conditions in the space environment include exposure to extreme heat and cold cycling, ultra-vacuum, atomic oxygen, and high energy radiation. Therefore, for the spatial environment, the substrate must have chemical, thermal, and vacuum suitable properties.

For this proposal, the selected substrate was the Rogers TMM (Thermoset Microwave Materials) an appropriated substrate for satellite communication systems. This substrate is a 9.8 relative dielectric permittivity $(\epsilon), 0.002$ loss tangent (tan $(\delta)), 6.35 \mathrm{~mm}$ thickness (d), and has an exceptionally low coefficient of thermal expansion (CTE) of dielectric constant, typically less than $30 \mathrm{ppm} / \mathrm{C}$. These laminates have isotropic CTE, very closely matched to copper [22].

The geometry of the prototype was influenced by the design strategies of Huang [19] and Kakoyiannis [17]. The substrate size (Wg x Lg) was limited by $100 \mathrm{~mm}$ x 100 $\mathrm{mm}$ and the patch size (Wp $\mathrm{x}$ Lp) was arbitrated for 80 $\mathrm{mm} \times 80 \mathrm{~mm}$. The feeding point of the coaxial probe was chosen on the diagonal of the patch. Using the feeding on the diagonal line, two orthogonal resonant modes are excited and resulting a linearly polarized radiation at both frequencies. The appropriate feeding point was determined through a routine of optimization using the HFSS Genetic Algorithm looking for the best $S_{11}$ response for both frequencies.

Fig. 3 presents the final geometry. This approach has some differences concerning Kakoyiannis's work [17]. In this approach, coaxial probe power was used instead of the microstrip feed, this change reduces the total area of the antenna. Further, there is a difference in size between the slits at the edges parallel to the $\mathrm{x}$-axis concerning the edges parallel to the $\mathrm{y}$ axis, this asymmetry guarantees dual-band operation.

The length and width of each slit were determined empirically until the desired resonance frequencies were obtained. The optimization process of the antenna was performed by adjusting geometric parameters accordingly. In the first step, the number of slits was increased until obtaining frequencies close to the desired one. Later, the length and width of the slits were modified to obtain the fine adjustment of the resonance frequencies. The final measurements showed the slits with widths of $2 \mathrm{~mm}$ and equally spaced $2 \mathrm{~mm}$. Additional simulations were carried out to determine the radiation pattern and estimate the antenna gain and efficiency.

The manufacturing process starts printing the layout of the patch on the substrate using an inkjet printer, with ultraviolet technology capable of printing on substrates up to $50 \mathrm{~mm}$ thick. After the printing process, the etching procedure was carried out. Finally, the substrate was drilled with a $1.2 \mathrm{~mm}$ drill, and a $50 \Omega$ SMA connector was welded for feeding the RF signal. The Fig. 4 shows the prototype of the built antenna, after adjusting the dimensions of the stubs to ensure operation at the desired frequencies. 


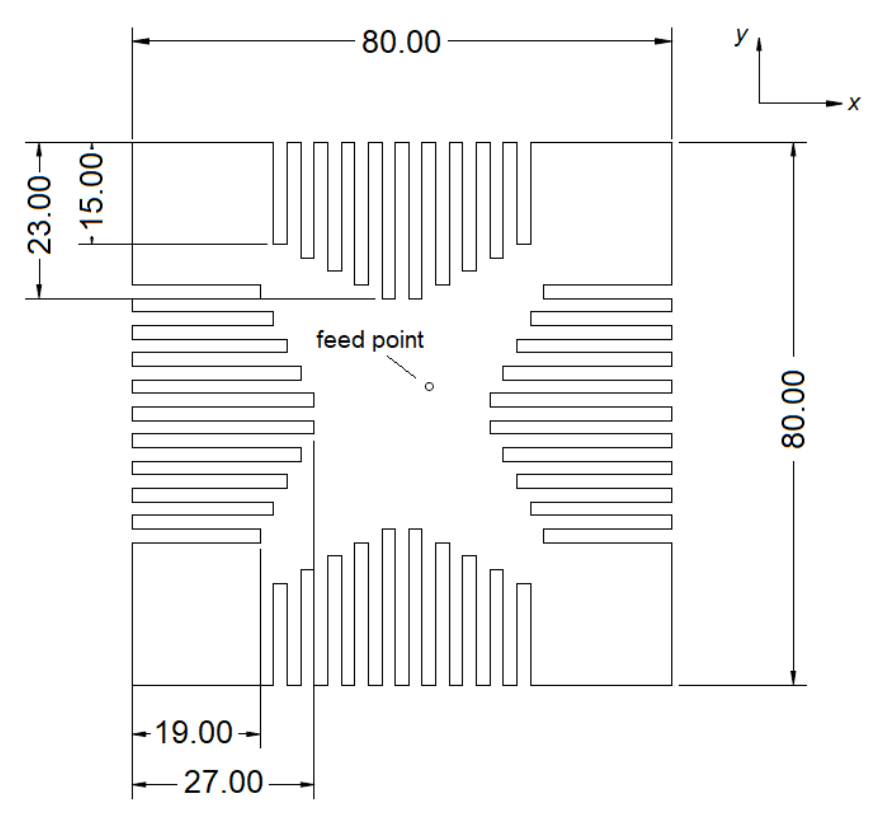

Fig. 3. The Final geometry of the microstrip patch antenna, dimensions in $\mathrm{mm}$.

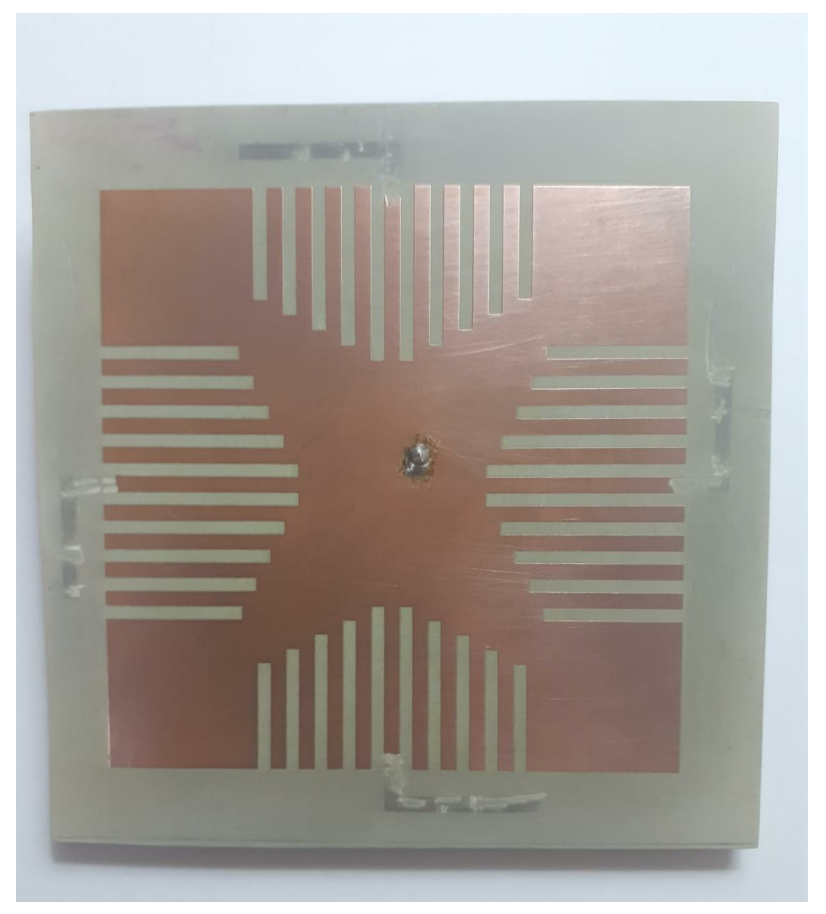

Fig. 4. The prototype of the proposed microstrip patch antenna.

The prototype $S_{11}$ measurements were performed using a Vector Network Analyzer (VNA), model N5230C Agilent Technologies, configured for a sweep from $350 \mathrm{MHz}$ to 500 $\mathrm{MHz}$ at $25 \mathrm{kHz}$ steps. It was not possible to measure the radiation parameters because the anechoic chamber available was small to measure in the far-field at the low UHF band. However, a detailed analysis was carried out using the HFSS program to obtain an adequate characterization of the antenna.

\section{RESULTS AND DISCUSSION}

Simulations and optimizations were performed for frequencies slightly below the desired frequencies. The purpose of this approach was to use stubs that could be gradually reduced, by mechanically removing the conductive patch material, and thereby increasing the frequency of operation. After optimizing the antennas geometric parameters, a dual-frequency response with very narrow impedance bandwidths can be obtained, in accord with Fig. 5. The best-found simulated magnitude of the $S_{11}$ was $-23.52 \mathrm{~dB}$ and $-17.47 \mathrm{~dB}$, at 400.975 $\mathrm{MHz}$ and $430.475 \mathrm{MHz}$ at central frequencies, respectively.

After the first measurements on the prototype, it was found that the operating frequencies were much lower than expected. Even completely removing the stubs it was not possible to reach the desired operating frequency in the lowest operating band. The final measured $S_{11}$ results were $-13.87 \mathrm{~dB}$ and - $10.05 \mathrm{~dB}$, at $398.9 \mathrm{MHz}$ and $431.4 \mathrm{MHz}$ frequencies, respectively. One can design the antenna to a slightly higher frequency by adjusting the length of the slits. However, due to restrictions imposed by the COVID-19 pandemic, it was not possible to build a new prototype.

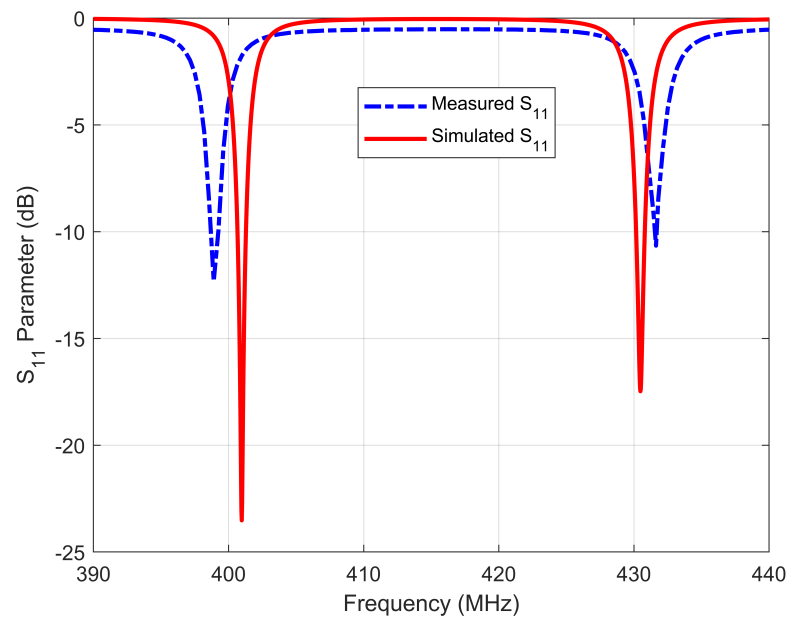

Fig. 5. Simulated and measured magnitude of the $S_{11}$ parameter after optimizing process.

Fig. 6 shows the Smith chart for simulated and measured input impedance characteristics. The measured impedance bandwidths, considering a VSWR=3:1 $\left(\left|S_{11}\right|<6 \mathrm{~dB}\right)$ for both frequencies, are close to $0.30 \%$. Therefore, the antenna exhibits a very narrow bandwidth, and it is in accordance with the theoretical prediction.

Input resistance $\left(R_{i n}\right)$ and input reactance $\left(X_{i n}\right)$ values measured from the network analyzer were plotted as a function of frequency, Fig 7. Impedance measurements show that the antenna has a highly inductive behavior, this is due to coaxial probe feeding. The input impedance presented values of 34.60 $+\mathrm{j} 13.88 \Omega$ and $75.42+\mathrm{j} 37.75 \Omega$ for frequencies of $398.9 \mathrm{MHz}$ and $431.4 \mathrm{MHz}$, respectively.

The radiation pattern can be considered as being driven by the induced surface current density on the patch element in the presence of the grounded dielectric substrate [23]. Fig. 8 presents the surface current distribution for both frequencies. 


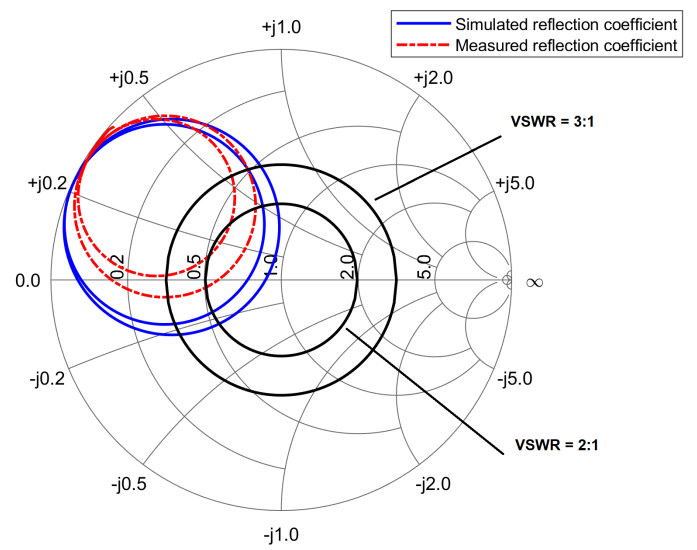

Fig. 6. Smith chart for simulated and measured input impedance characteristics.

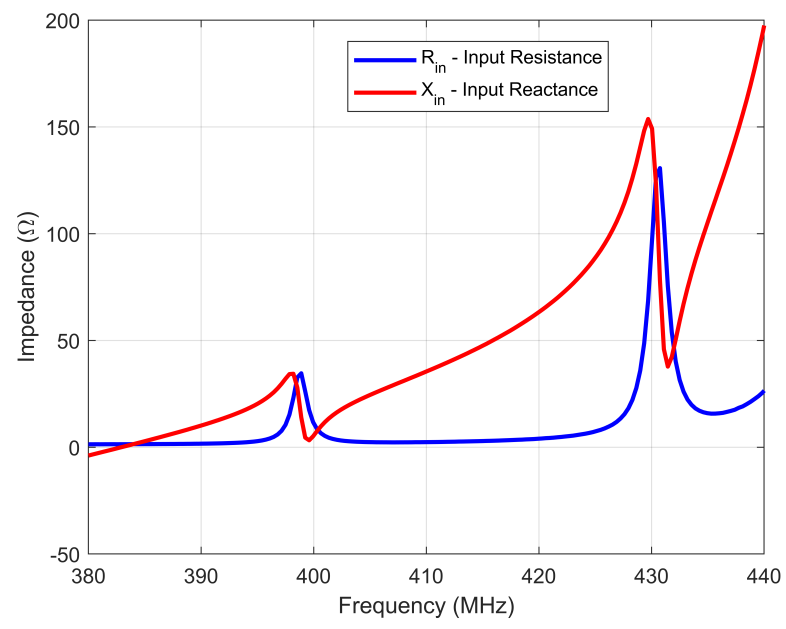

Fig. 7. Measured input resistance $\left(R_{i n}\right)$ and input reactance $\left(X_{i n}\right)$.

Typical directivity is $7.0 \mathrm{~dB}$ for a microstrip patch antenna and it presents a broadside pattern. However, in the presence of a ground plane of dimensions smaller than $/ 2$, the radiation pattern may present a directivity close to $2.2 \mathrm{~dB}$ with an omnidirectional pattern. The radiation patterns for the operating frequencies were simulated and presented omnidirectional patterns close to a half-wave dipole, as illustrated by the threedimensional and two-dimensional radiation patterns in Fig. 9 and Fig. 10, respectively.

Considering the values of the electrical size (ka) of the antenna, the maximum theoretical gain estimated by (1) is $1.87 \mathrm{~dB}$ and $2.25 \mathrm{~dB}$ for $400.975 \mathrm{MHz}$ and $430.475 \mathrm{MHz}$, respectively. The simulated directivities were $2.62 \mathrm{~dB}$ and 2.81 $\mathrm{dB}$, for $400.975 \mathrm{MHz}$ and $430.475 \mathrm{MHz}$, respectively. The maximum directivity is in the z-axis direction, which leads us to the conclusion that the best direction for pointing the antenna will be the z-axis direction. This pointing direction ensures the maximum gain in both operating frequencies. In addition, the antenna exhibits nulls in the $\mathrm{x}$-axis and $\mathrm{y}$-axis directions at $400.975 \mathrm{MHz}$ and $430.475 \mathrm{MHz}$, respectively.

The simulated AR (axial ratio) is shown in Fig. 11 to both
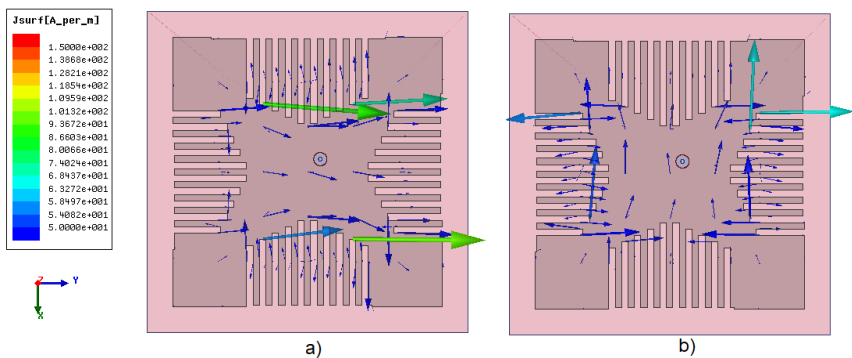

Fig. 8. The simulated surface current distribution at (a) $400.975 \mathrm{MHz}$ and (b) $430.475 \mathrm{MHz}$

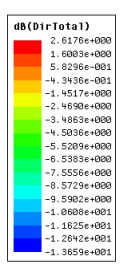

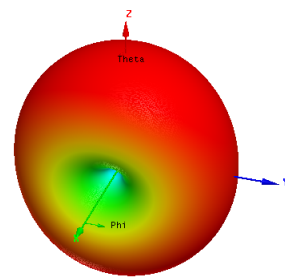

(a)
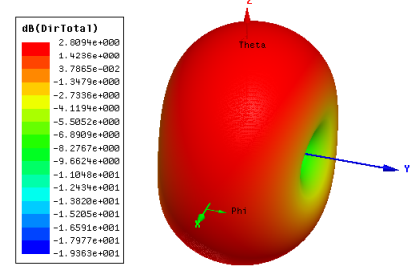

(b)
Fig. 9. The simulated three-dimensional radiation pattern of the antenna at (a) $400.975 \mathrm{MHz}$ and (b) $430.475 \mathrm{MHz}$.

frequencies. The AR is the ratio of the major to minor axes of a polarization ellipse [24]. Simulated results are above 30 $\mathrm{dB}$ across the entire range of $\theta$, at $\phi 90$ and 0 , at 400.975 $\mathrm{MHz}$ and $430.475 \mathrm{MHz}$, respectively. Circular polarization is theoretically characterized by an AR close to $3 \mathrm{~dB}$. From the Fig. 11, it can be found that the simulated AR is greater than $30 \mathrm{~dB}$, this leads to the conclusion that the polarization is not circular.

In addition to $\mathrm{AR}$, the simulations to both co-polarized and cross-polarized radiation patterns were performed in $\mathrm{xz}$ plane at $400.975 \mathrm{MHz}$ and in yz plane at $430.475 \mathrm{MHz}$, the antenna is in the xy plane, according to Fig. 12. The radiation patterns show good monopole-type radiation for the E-planes with good cross polar discrimination of at least $-30 \mathrm{~dB}$.

Using the feeding point in the patch diagonal, two orthogonal resonant modes are excited resulting that the linearly polarized radiation is also orthogonal at two frequencies. Therefore, the antenna presents linear polarization for both frequencies at the $z$ direction of the propagation. Considering that the antenna embedded in the satellite presents a relative movement in relation to the earths surface, it is necessary that the ground station has a circularly polarized antenna to guarantee reception at both frequencies regardless of the linear polarization of the satellite antenna, whether horizontal or vertical.

Table II summarizes simulated and measured main performance parameters. Despite its low gain and narrow bandwidth, this proposal meets the requirements of CONASAT project since a low transmission rate (9600 bps) is expected and the gain can be offset by the ground system. 

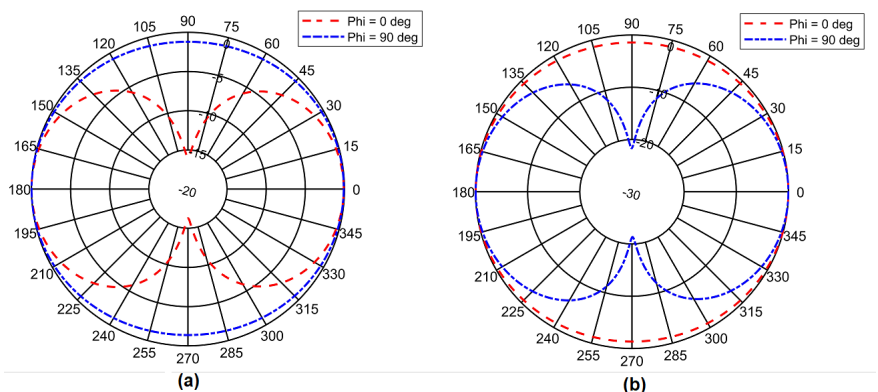

Fig. 10. Radiation pattern of the antenna at (a) $400.975 \mathrm{MHz}$ and (b) 430.475 MHz.

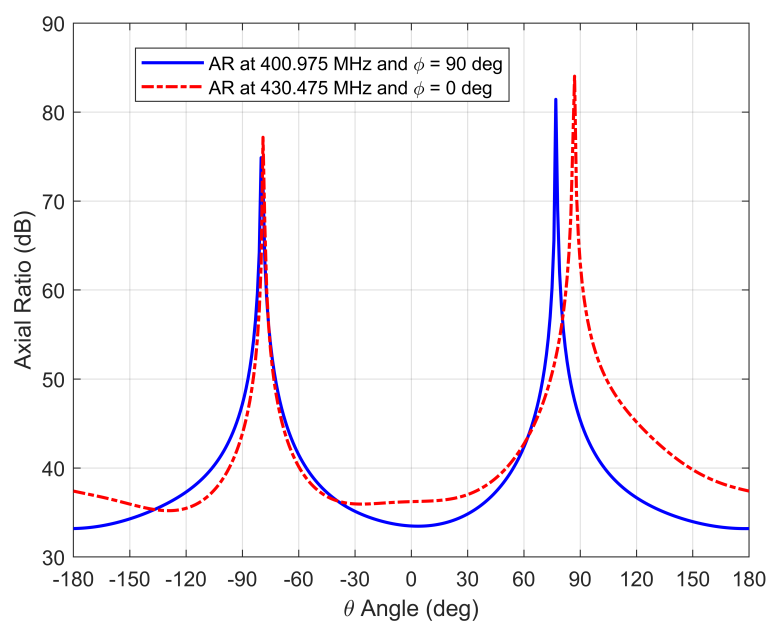

Fig. 11. The simulated axial ratio of the antenna.

TABLE II

SIMULATED AND MEASURED PERFORMANCE PARAMETERS

\begin{tabular}{lcc}
\hline \multicolumn{1}{c}{ Parameters } & Simulations & Measurements \\
\hline Freq. (MHz) & $400.975 / 430.475$ & $398.9 / 431.4$ \\
$\left|S_{11}\right|(\mathrm{dB})$ & $23.52 / 17.47$ & $13.876 / 10.05$ \\
Gain $(\mathrm{dB})$ & $-3.52 /-2.23$ & - \\
Directivity (dB & $2.62 / 2.81$ & - \\
Efficiency (\%) & $24.33 / 31.33$ & - \\
BW (MHz) & $1.100 / 1.175$ & $1.225 / 1.125$ \\
BW (\%) & $0.27 / 0.27$ & $0.30 / 0.26$ \\
ka & $0.5938 / 0.6375$ & $0.5909 / 0.6391$ \\
\hline
\end{tabular}

The simulated efficiency was calculated from the ratio of simulated gain to simulated directivity. This was estimated close to $24 \%$ and $31 \%$ at $400.975 \mathrm{MHz}$ and $430.475 \mathrm{MHz}$, respectively. The efficiency of the proposed antennas is relatively low and it imposes some limitations on the application of the proposed slitted antennas. However, this can be overcome considering the optimization for ground-to-space communication link.

\section{CONCLusions}

This study aimed to design, simulate, manufacture, and measure a dual-band microstrip patch antenna operating in the UHF band and meeting the restrictions for a possible application in the Brazilian CubeSat CONASAT program. Besides a literature review on small antennas and CubeSat
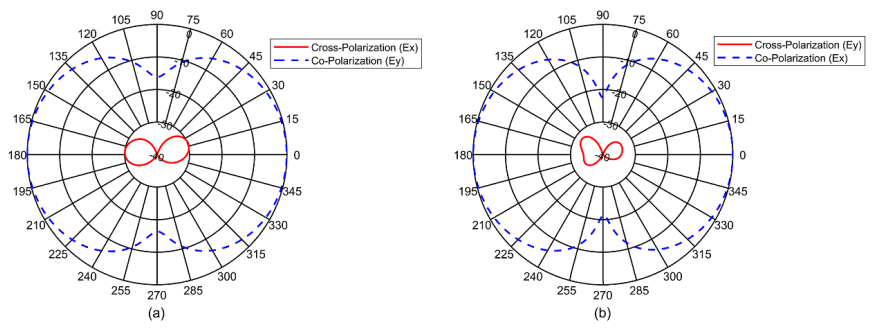

Fig. 12. The simulated co-polarized and cross-polarized radiation patterns for a) $\mathrm{xz}$ plane at $400.975 \mathrm{MHz}$, and b) yz plane at $430.475 \mathrm{MHz}$.

antennas is presented. A prototype of the designed antenna was realized. Forty slits, on the perimeter of a square patch, ten slits on each edge, and a high dielectric constant substrate was used to reduce the operating frequencies. The proposed antenna resonates close to $400 \mathrm{MHz}$ and $431 \mathrm{MHz}$ frequencies and it is limited at $100 \mathrm{~mm} \times 100 \mathrm{~mm}$ area in a CubeSat face. Simulations and experimental measurements are in accord and confirm that the antenna presents suitable operating frequencies for a CubeSat application. We have found an innovative approach that presents a dual-band with a single feeding point. This work presents its contribution since it makes it possible for two antennas to be replaced by one. The patch antennas reduce the risks inherent to deployable wire antennas. In this way, this proposal reduces weight, volume, costs, risks and increasing the reliability of the small satellite telecommunications system. It is well known that conventional Microstrip Patch Antennas (MPA) has some drawbacks as low efficiency and narrow bandwidth. These disadvantages become critical for compact antennas. Therefore, for future works, it could be interesting to consider other geometries and materials to obtain better efficiency and bandwidth besides circular polarization. For example, the use of metamaterials can be an interesting line of research. The proposal presented in this work is not restricted to the CONASAT program, but it can be useful for several programs that use small satellites since the operating frequencies are widely used in most similar projects.

\section{ACKNOWLEDGMENTS}

The authors would like to thank Dr. Daniel Chagas (ITA Aeronautics Institute of Technology) and Eng. Julio Cesar (IFI - Industrial Promotion and Coordination Institute) for useful discussions and for support in measurement process. We also thank M. Sc. Colonel Luciano Magalhes (Director of the IFI) for allowing the use of the facilities at IFI.

\section{REFERENCES}

[1] CubeSat, "Cubesat design specification," tech. rep., 2014.

[2] J. Coopersmith, "Affordable Access to Space." https://issues.org/jonathan/, 2012. [Issues in Science and Technology 29, no. 1 (Fall 2012). ISSN: 0748-5492. Online; accessed 29-May-2019].

[3] T. Alam, M. Islam, and M. Cho, "Near-zero metamaterial inspired uhf antenna for nanosatellite communication system," Nature: Scientific Reports, vol. 9, pp. 1-15, March 2019, DOI:10.1038/s41598-019-402073.

[4] E. A. M. de Souza, Antenas Compactas e Miniaturizadas para Sistemas de Comunicacao sem Fio e Nanossatelites do Padrao CubeSat. Phd thesis (in portuguese), Universidade Federal do Rio Grande do Norte, 2018. 
[5] S. Gao and et al., "Antennas for small satellites," Loughborough Antennas \& Propagation Conference, Loughborough, UK, pp. 66-69, 2008.

[6] J. Verwilligen and P. Sundaramoorthy, "A Novel Planar Antenna for CubeSats," 29th Annual AIAA/USU - Conference on Small Satellites, pp. 1-8, 2015

[7] W. L. Stutzman and G. A. Thiele, Antenna Theory and Design. John Wiley \& Sons, Inc., 3rd ed., 2012.

[8] J. L. Volakis, C.-C. Chen, and K. Fujimoro, Small Antennas: Miniaturization Techniques \& Applications. New York: McGraw-Hill, 1st ed., 2010.

[9] L. Huitema and T. Monediere, Compact Antennas An overview, pp. 123. Progress in Compact Antennas, Laure Huitema (Ed.), Vienna, Austria: InTech, 2014.

[10] A. H. Lokman and et al., "A Review of Antennas for Picosatellite Applications," International Journal of Antennas and Propagation, vol. 2017, 2017, DOI: $10.1155 / 2017 / 4940656$.

[11] H. A. Wheeler, "Fundamental Limitations of Small Antennas," Proceedings of the IRE, vol. 35, no. 12, pp. 1479-1484, 1947, DOI: 10.1109/JRPROC.1947.226199.

[12] L. J. Chu, "Physical Limitations of Omnidirectional Antennas," Journal of Applied Physics, vol. 19, pp. 1163-1175, 1948, DOI: 10.1063/1.1715038.

[13] A. Skrivervik, J. Zurcher, O. Staub, and J. Mosig, "PCS antenna design: the challenge of miniaturization," IEEE Antennas Propag. Mag., vol. 43, no. 4, pp. 12-27, 2001, DOI: $10.1109 / 74.951556$

[14] R. F. Harrington, "Effect of antenna size on gain, bandwidth, and efficiency," Journal of Research of the National Bureau of Standards, vol. 64D, pp. 1-12, January- February 1960.

[15] B. Klofas, J. Anderson, and K. Leveque, "CubeSats in Detail: A Survey of CubeSat Communication Systems," The AMSAT Journal, pp. 23-30, December.

[16] B. Klofas and K. Leveque, "A survey of cubesat communications systems: 2009-2012,” 2013. In: CalPoly CubeSat Developers Workshop, 2013.

[17] C. Kakoyiannis and P. Constantinou, Electrically Small Microstrip Antennas Targeting Miniaturized Satellites: the CubeSat Paradigm, pp. 273-316. Microstrip Antennas, Prof. Nasimuddin Nasimuddin (Ed.), Vienna, Austria: InTech, 2011.

[18] Y. Rahmat-Samii, V. Manohar, and J. M. Kovitz, "For satellites, think small, dream big: A review of recent antenna developments for cubesats.," IEEE Antennas Propag. Mag., vol. 59, pp. 22-30, April 2017, DOI: 10.1109/MAP.2017.2655582.

[19] J. Huang, "Miniaturized uhf microstrip antenna for a mars mission," IEEE Antennas and Propag. Society International Symposium, pp. 486 489, July 2001, DOI: 10.1109/APS.2001.959504.

[20] R. Garg, P. Bhartia, I. Bahl, and A. Ittipiboon, Microstrip Antenna Design Handbook. Boston: Artech House, 2001.

[21] D. M. Pozar, A Review of Bandwidth Enhancement Techniques for Microstrip Antennas, pp. 157-166. Microstrip Antennas - The Analysis and Design of Microstrip Antennas and Arrays, David M. Pozar and Daniel H. Schaubert (Ed.), New York: IEEE Press, 1995.

[22] Rogers, "TMM Laminate." https://www.rogerscorp.com/, 2019. [Online; accessed 09-April-2019].

[23] D. M. Pozar, Microwave Enginneering. New York: JohnWiley \& Sons, 4th ed., 2011.

[24] IEEE, "IEEE Standard for Definitions of Terms for Antennas," tech. rep., IEEE Antennas and Propag. Society, New York, 2013.

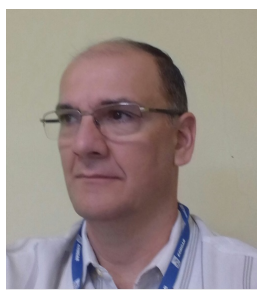

Wellington Guilherme da Silva was born in Rio de Janeiro, RJ, Brazil in 1969. He received the B.S degree in electrical engineering from the University of Pernambuco, Recife, Brazil, in 2000 and M.S in Physics degree from the Aeronautics Institute of Technology, São José dos Campos, SP, Brazil, in 2010. He is currently working toward the Ph.D. degree at Federal University of Rio Grande do Norte, Natal RN, Brazil.

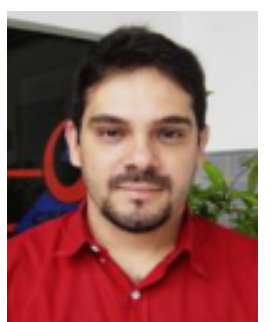

Antonio Luiz P. S. Campos was born in Natal, Brazil in 1972. He received the B.S. and M.S. degrees in electrical engineering from the Federal University of Rio Grande do Norte, Natal RN, Brazil, in 1996 and 1999, respectively, and the Ph.D. degree in electrical engineering at the Federal University of Paraíba, Campina Grande PB, Brazil, in 2002. Dr Campos is currently involved in the areas of FSS, scattering of electromagnetic waves, antennas, and planar devices. He is a member of the Brazilian Microwave and Optoelectronics Society (SBMO).

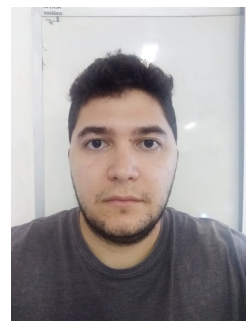

monitoring systems.
Juan Rafael Filgueira Guerra was born in Mossor, Brazil in 1992. He received the B.S. degree in energy engineering in 2014 and M.S. degree in electrical engineering in 2016, both from the Federal Rural University of Semi-Arid, Mossor RN, Brazil. Since 2017, he has been working towards the Ph.D. degree at the Postgraduate Program in Electrical and Computer Engineering (PPGEEC) in Technology Center, Federal University of Rio Grande do Norte, Natal, Brazil. His research is about applying FSS and antennas on technologies to structural health 